\title{
Histoire de la pensée - et ce qui change à l'ère numérique
}

\section{Jean Martin}

Dr méd., membre de la rédaction

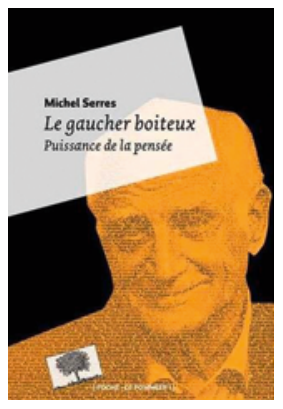

Michel Serres

Le gaucher boiteux

Puissance de la pensée

Paris: Editions Le Pommier; 2014, réédition 2017.

280 pages. $15.30 \mathrm{CHF}$.

ISBN 978-2746506954

Encore un livre du philosophe des sciences Michel Serres, pièce d'une trilogie dont j'ai déjà parlé $[1,2]-$ Serres, «un des rares philosophes contemporains à proposer une vision du monde qui associe les sciences et la culture». Cet ouvrage investigue l'évolution de la pensée et des connaissances, dans le cadre du Grand Récit de l'histoire de l'univers; "relatant l'apparition de phénomènes rares, imprévisibles, contingents; apparition d'éléments, productions de figures, inventions». Un mot sur le titre surprenant: les savants du XIX $\mathrm{X}^{\mathrm{e}}$ siècle ouvrirent «le règne du chaos et de la contingence, l'instabilité de notre monde, boiteux et gaucher» - mais l'auteur dit aussi cela de plusieurs héros antiques.

Le propos est développé à la façon Serres: langage fleuri, feu d'artifice qui peut laisser haletant, nourri de l'immense érudition de l'auteur: savoir scientifique aussi bien que philosophique, culture à la française. On aimerait parfois quelque chose d'un peu resserré, qui limite les détours ou digressions (en dépit de leur utilité) voire les envolées du registre poétique, pour avancer un peu plus vite vers l'enseignement voulu. Mais le style c'est l'homme... Si j'ose des termes de météo, on vit/lit sous des bouffées de vent, des éclats de soleil, des éclairs parfois, au bord d'eaux fluides ou torrentueuses...

Comme dans ses autres livres, réflexions larges, pas rarement bousculantes, sur notre époque (celle de sa petitefille Petite Poucette, «née lorsque ordinateur et téléphone portable changèrent nos relations et notre cognition»). Serres en est convaincu: on est dans l'ère d'Hermès, le messager, le communicateur, plus dans celle de Mars. Parlant ici à nouveau de l'entrée dans un «âge doux». Des extraits en donnent une idée.

"Qu'est-ce que penser? Au minimum effectuer ces quatre opérations; recevoir, émettre, stocker, traiter de l'information" - par quoi l'homme a avancé au cours des millénaires, et qui sont les mêmes opérations que réalisent les nouveaux moyens informatiques d'aujourd'hui.

Evolution: «La vie opère par émergences, par synthèses inattendues, elle explose d'inventions. Comme le cosmos avec son expansion, l'évolution peut passer pour une fabrique de nouveautés.» Changements sociétaux. «Nos techniques se découplent de cette ancienne attache, où régnaient la séparation des sujets pensants (et puissants) et des objets (humains) passifs à exploiter pour s'accoupler aux sciences de la vie et de la Terre où foisonne le possible, aussi bien qu'à celles de l'information, où règne le virtuel.» Il lui paraît qu'«agonise l'ère où la concentration faisait le pouvoir, le savoir la fortune, l'Etat, la société». Peut-être... on espère que ces effets bénéfiques se concrétiseront.

«Cruciale, la bataille sociale et politique d'aujourd'hui concerne les données (disponibles en masse), puisque peu à peu elles équivaudront à l'argent (...) Voilà désormais le bien commun, bien de tous et de chacun, souvent de manière difficile à partager.» Universelle, la transparence "laisse certes aux puissants la possibilité de développer des surveillances et d'abominables espionnages. Mais, aussi, un seul individu peut dénoncer aussitôt ces abus et rendre les coupables détestables universellement» - rappel de ce qu'Esope dit de la langue, la meilleure et la pire des choses.

A propos de nature et d'environnement. «La construction de l'identité ne procède pas seulement de l'entourage humain mais aussi, peut-être surtout, des eaux, plantes et bêtes. L'existence exclusivement humaine et politique handicape à tel point que la majorité de nos contemporains rapetissent de manière infantile.» «Cette classe ou cathédrale buissonnière [la nature] construit l'ego des ados - j'ai vécu cela comme eux - au moins autant que l'école ou la famille.»

Le but. «Je voudrais, avant de mourir, devenir sagefemme, aider à l'enfantement du nouveau monde. Ma vie entière m'y a préparé, par l'écoute attentive des craquements émis par l'ancien. Les crises que nous traversons, je les entends comme des plaintes émises en travail de gésine.» C'est dire que, si Serres s'approche de 90 ans, nous devons néanmoins nous attendre à le lire encore. 